\title{
The cytolethal distending toxin from the chancroid bacterium Haemophilus ducreyi induces cell-cycle arrest in the G2 phase
}

\author{
Ximena Cortes-Bratti, ${ }^{1}$ Esteban Chaves-Olarte, ${ }^{1}$ Teresa Lagergård, ${ }^{2}$ \\ and Monica Thelestam ${ }^{1}$ \\ ${ }^{1}$ Microbiology and Tumorbiology Center, Karolinska Institutet, S-171 77 Stockholm, Sweden \\ ${ }^{2}$ Department of Medical Microbiology and Immunology, University of Gothenburg, S-413 46 Gothenburg, Sweden \\ Address correspondence to: Monica Thelestam, Microbiology and Tumorbiology Center, Karolinska Institutet, Box 280, S-171 77 \\ Stockholm, Sweden. Phone: 46-8-728 71 62; Fax: 46-8-33 15 47; E-mail: Monica.Thelestam@mtc.ki.se \\ Received for publication April 27, 1998, and accepted in revised form November 13, 1998.
}

\begin{abstract}
The potent cytolethal distending toxin produced by Haemophilus ducreyi is a putative virulence factor in the pathogenesis of chancroid. We studied its action on eukaryotic cells, with the long-term goal of understanding the pathophysiology of the disease. Intoxication of cultured human epithelial-like cells, human keratinocytes, and hamster fibroblasts was irreversible, and appeared as a gradual distention of three- to fivefold the size of control cells. Organized actin assemblies appeared concomitantly with cell enlargement, promoted by a mechanism that probably does not involve small GTPases of the Rho protein family. Intoxicated cells did not proliferate. Similar to cells treated with other cytolethal distending toxins, these cells accumulated in the G2 phase of the cell cycle, demonstrating an increased level of the tyrosine phosphorylated (inactive) form of the cyclin-dependent kinase $\mathrm{p} 34^{\mathrm{cdc} 2}$. DNA synthesis was not affected until several hours after this increase, suggesting that the toxin acts directly on some kinase/phosphatase in the signaling network controlling the $\mathrm{p} 34^{\mathrm{cdc} 2}$ activity. We propose that this toxin has an important role both in the generation of chancroid ulcers and in their slow healing. The toxin may also be an interesting new tool for molecular studies of the eukaryotic cell- cycle machinery.
\end{abstract}

J. Clin. Invest. 103:107-115 (1999).

\section{Introduction}

Haemophilus ducreyi is a Gram-negative, facultatively anaerobic coccobacillus that causes chancroid (soft chancre). This sexually transmitted disease develops with characteristic mucocutaneous, shallow ulcers on the external genitals. The ulcers are painful and show a pronounced retardation of healing. The disease is endemic in many developing countries, where an association between genital ulcers and transmission of HIV has been demonstrated (1-4). Information is limited concerning the pathogenic mechanisms responsible for the development of ulcers in chancroid. A few bacterial components potentially involved in destruction of cells and tissues have been identified. The lipo-oligosaccharide caused a dermal lesion in animal models $(5,6)$, but the concentrations needed were unphysiologically high. The $122-\mathrm{kDa}$ cell-associated hemolysin was able to kill human foreskin fibroblasts in vitro $(7,8)$, but a hemolysin-negative isogenic mutant was equally active as the wild type in causing pustules in human skin (9).

A separate, secreted cytotoxin of $H$. ducreyi was shown to kill cultured epithelial-like cells of human origin (10). Immunization of rabbits with live bacteria or bacterial products elicited toxin-neutralizing serum antibodies (11). The toxin produced by different $H$. ducreyi strains was immunologically similar. A neutralizing monoclonal antibody (MAB M4D4) was prepared and used for immunoaffinity purification (12), yielding a cytotoxic $20-\mathrm{kDa}$ protein that shared the same or similar epitope for all toxic strains (12). This $H$. ducreyi toxin induced cell enlargement followed by cell death (10). The effect is similar to that of the cytolethal distending toxins (CDTs) encoded by a cluster of three linked genes $(c d t A, B, C)$ that have been detected in Escherichia coli (CDT-I, CDT-II, CDT-III) (13-15), Shigella (16), Campylobacter (17), and Actinobacillus actinomycetemcomitans (18).

Recently, the H. ducreyi cytotoxin was shown to be encoded by a similar cluster of three linked genes (19). Henceforth in this paper, it will be referred to as the $H$. ducreyi cytolethal distending toxin (HdCDT). The three proteins have calculated molecular masses of 25,30 , and $20 \mathrm{kDa}$ and are $38 \%, 51 \%$, and $24 \%$ identical with the products of the E. coli genes encoding CDT-I (13). HdCDT is even more related to the Actinobacillus CDT: the identities between the predicted amino acid sequences are $91 \%, 97 \%$, and $94 \%$ (18). To our knowledge, HdCDT is the only one of the CDTs known to date that has been purified. The $\mathrm{NH}_{2}$-terminal amino acid sequence of the purified HdCDT (12) showed 100\% homology with the $\mathrm{NH}_{2}$-terminal sequence of the cloned $20-\mathrm{kDa} c d t C$ protein product (19). The HdCDTneutralizing MAB M4D4 (12) bound to a His-fusion involving the $20-\mathrm{kDa} c d t \mathrm{C}$ protein product (19). These findings suggest that the active site of the toxin is localized in the C protein (19). Nonetheless, all three $H$. ducreyi cdt genes had to be expressed in recombinant $E$. coli strains to produce cytotoxic culture supernatants (19). Similar findings have been reported for the E. coli 

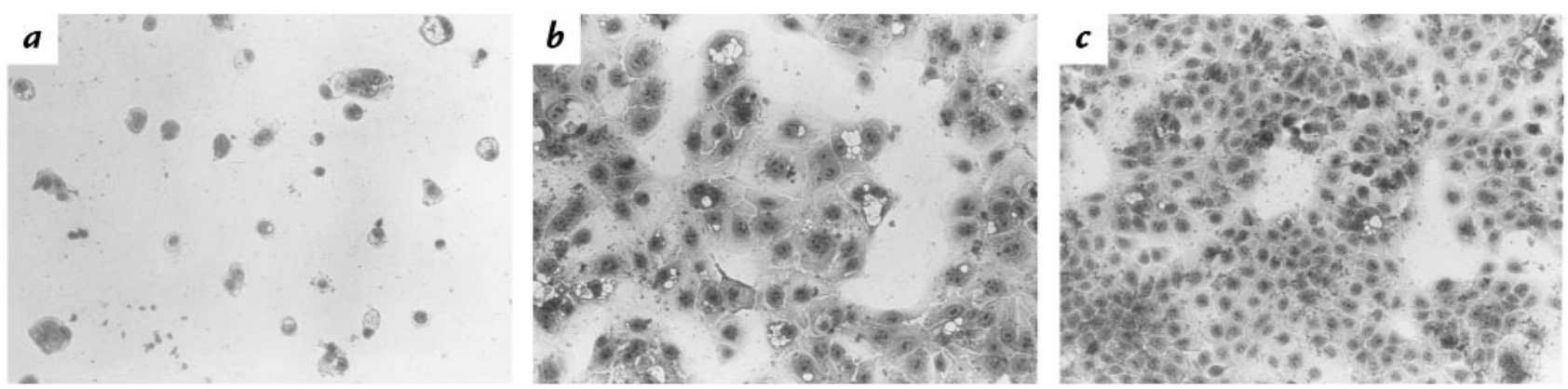

Figure 1

Effect of HdCDT on HaCat keratinocytes. Growing cells were exposed to $122 \mathrm{ng} / \mathrm{ml} \mathrm{HdCDT} \mathrm{for} 2 \mathrm{~h}$ at $37^{\circ} \mathrm{C}$. After postincubation in medium for 48 $\mathrm{h}(\boldsymbol{a})$ or $24 \mathrm{~h}(\boldsymbol{b})$, cells were fixed and stained with Giemsa. (c) Control cells, $48 \mathrm{~h} . \times 100$. HdCDT, Haemophilus ducreyi cytolethal distending toxin.

CDTs, but the roles of the $c d t A$ and $c d t B$ protein products are not yet clear $(13,15)$.

The E. coli, Campylobacter, and Actinobacillus crude CDTs have all been shown to induce cell-cycle arrest in the G2 phase $(15,18,20,21)$. A requirement for the transition of cells from G2 into mitosis is that the cyclin-dependent kinase $\mathrm{p} 34^{\mathrm{cdc} 2}$ is activated. The final step in that process is a dephosphorylation in Thr-14 and Tyr- 15 of $\mathrm{p} 34^{\mathrm{cdc} 2}$ (22). A hyperphosphorylation of $\mathrm{p} 34^{\mathrm{cdc} 2}$ has been reported to occur concomitantly with the cell-cycle arrest induced by CDTs $(20,21)$. Here we report that the purified HdCDT also induces G2 arrest and inactivation of $\mathrm{p} 34^{\mathrm{cdc} 2}$. The tyrosine phosphorylation of $\mathrm{p} 34^{\mathrm{cdc} 2}$ was the earliest detectable toxin-induced effect, occurring before HEp-2 cell enlargement and promotion of the actin cytoskeleton (ACSK). HdCDT did not act by adenosine diphosphate (ADP) ribosylation or glucosylation, and the ACSK effect appeared not to involve small guanosine triphosphatases (GTPases) of the Rho protein family. HdCDT is likely to play an important role in the development, persistence, and slow healing of the ulcerative lesions characteristic of chancroid.

\section{Methods}

Materials. HdCDT was purified by immunoaffinity chromatography as described (12). The protein concentration was $122 \mu \mathrm{g} / \mathrm{ml}$. Toxin B from Clostridium difficile (TcdB) and Lethal Toxin from Clostridium sordellii (TcsL) were kindly supplied by C. von Eichel-Streiber (University of Mainz, Mainz, Germany). Clostridium botulinum exoenzyme C3 was from Upstate Biotechnology Inc. (Lake Placid, New York, USA). UDP- $\left[{ }^{14} \mathrm{C}\right]$ glucose (specific activity $318 \mathrm{mCi} / \mathrm{mmol}$ ) and [ $\left.{ }^{32} \mathrm{P}\right] \mathrm{NAD}$ (specific activity $800 \mathrm{Ci} / \mathrm{mmol}$ ) were from Du Pont Nen (Dreieich, Germany). Methyl- $\left[{ }^{3} \mathrm{H}\right]$ thymidine (specific activity $5.0 \mathrm{Ci} / \mathrm{mmol}$ ) and $[\gamma-$ $\left.{ }^{32} \mathrm{P}\right]$ ATP (specific activity $30 \mathrm{Ci} / \mathrm{mmol}$ ) were from Amersham Sweden AB (Solna, Sweden). All other reagents were of analytical grade and obtained from local commercial sources.

Cell culture and toxin treatment. Human larynx carcinoma cells (HEp-2; American Type Culture Collection [ATCC] No. CCL-23), Chinese hamster lung fibroblasts (Don; ATCC No. CCL-16), human cervix carcinoma cells (HeLa; ATCC No. CCL-2), and a human keratinocyte line (HaCaT; kindly provided by N.E. Fusenig, Heidelberg, Germany) (23) were cultivated in Eagle's MEM supplemented with $10 \% \mathrm{FBS}, 5 \mathrm{mM} \mathrm{L-}$ glutamine, penicillin $(100 \mathrm{U} / \mathrm{ml})$, and streptomycin $(100 \mu \mathrm{g} /$ $\mathrm{ml}$ ) in a humid atmosphere containing $5 \% \mathrm{CO}_{2}$. Mouse Swiss fibroblasts (3T3; ATCC No. CCL-92) were cultivated in DMEM with supplements as already described here. HEp-2 or
HeLa cells in logarithmic growth phase (in 96-well or 24-well plates) were cooled on ice for $15 \mathrm{~min}$ before addition of icecold toxin $(122 \mathrm{ng} / \mathrm{ml})$. After 15-min exposure, the cells were washed three times with cold HBSS. Eagle's MEM was added and the cells were further incubated at $37^{\circ} \mathrm{C}$. Don and $3 \mathrm{~T} 3$ fibroblasts were exposed to $122 \mathrm{ng} / \mathrm{ml} \mathrm{HdCDT}$ continuously at $37^{\circ} \mathrm{C}$. $\mathrm{HaCaT}$ cells suspended in culture medium were treated with $\mathrm{HdCDT}$ for $2 \mathrm{~h}$ at $37^{\circ} \mathrm{C}$ followed by toxin-free growth medium as described previously (10). The cytopathic effect (CPE) was neutralizable by preincubation of the toxin ( $30 \mathrm{~min}$ at $37^{\circ} \mathrm{C}$ ) with the MAB M4D4 (12).

Staining of F-actin with FITC-phalloidin and measurement of cell area. HdCDT-treated cells were stained with FITC-phalloidin and visualized by fluorescence microscopy as previously described (24). Ten randomly chosen fields were photographed and the negatives scanned (Personal Densitometer SI; Molecular Dynamics, Sunnyvale, California, USA). The cell areas were quantified using ImageQuant software (Molecular Dynamics).

Assay of small GTPases. Small GTPases in cell lysates were labeled with the glucosyltransferase toxins TcdB and TcsL in the presence of UDP- $\left[{ }^{14} \mathrm{C}\right]$ glucose. The GTPases were separated by SDS-PAGE and visualized by PhosphorImager (Molecu-

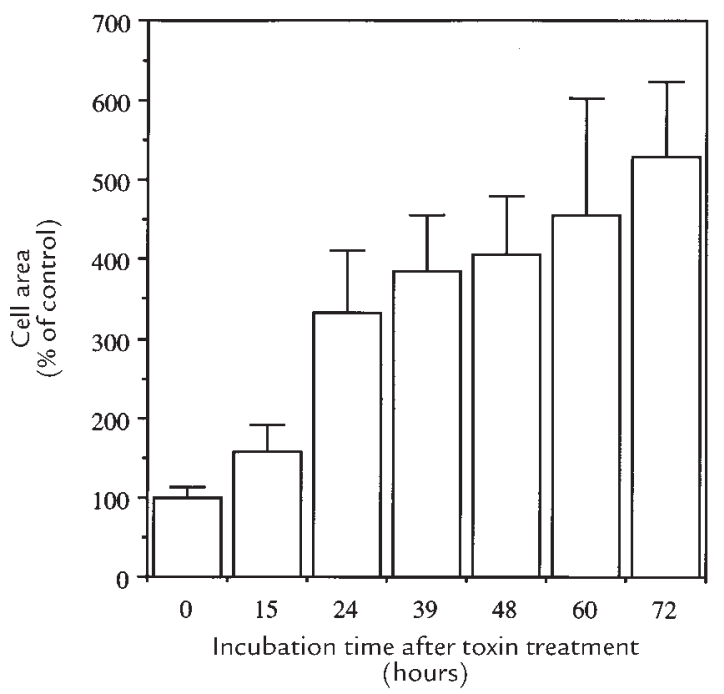

\section{Figure 2}

Area of HdCDT-treated HEp-2 cells. Cells were treated with HdCDT (15 min on ice). After postincubation at $37^{\circ} \mathrm{C}$ for the times indicated, cells were stained with FITC-phalloidin and photographed. Cell areas were quantified using ImageQuant software, and the results were expressed as percentage + SD of untreated control cells. 
lar Dynamics) analysis as described (25). Cell lysates were sonicated three times for $3 \mathrm{~s}$, centrifuged $(3,500 \mathrm{~g} \times 10 \mathrm{~min})$, and the supernatants were subjected to high-speed centrifugation $(52,000 \mathrm{~g} \times 1 \mathrm{~h})$ at $8^{\circ} \mathrm{C}$. The supernatants from the second centrifugation were used as cytosol. The pellets, resuspended in $200 \mu \mathrm{l}$ of lysis buffer, were used as the membrane fraction.

Assay of ADP-ribosyltransferase activity and glucosyltransferase activity. ADP-ribosyltransferase activity was assayed as described (24) by incubating HdCDT $(12.2 \mathrm{~g} / \mathrm{ml})$ with fresh cell lysate and [ $\left.{ }^{32} \mathrm{P}\right] \mathrm{NAD}$ for $60 \mathrm{~min}$, followed by SDS-PAGE of the proteins and detection of radioactivity. The C. botulinum ADP-ribosyltransferase C3 served as positive control. Glucosyltransferase activity was assayed as described (25), with TcdB as positive control.

Assay of DNA synthesis. DNA synthesis was assayed by two methods: (a) Toxin-treated HEp- 2 cells were incubated at $37^{\circ} \mathrm{C}$ in medium for 6,12 , and $24 \mathrm{~h}$ followed by fresh medium with $10 \mu \mathrm{g} / \mathrm{ml}$ bromodeoxyuridine (BrdU; Sigma Chemical Co., St. Louis, Missouri, USA) for $12 \mathrm{~h}$. Cells were fixed and permeabilized for immunofluorescence (24) and incubated (90 $\mathrm{min})$ in a humid chamber with monoclonal anti-BrdU (Clone BU-33; Sigma Chemical Co.) diluted 1:1,000 in PBS containing 0.5 $\mathrm{mg} / \mathrm{ml}$ DNAse I (Sigma Chemical Co.) and $0.1 \%$ BSA. After two rinses with PBS and incubation $\left(60 \mathrm{~min}\right.$ at $22^{\circ} \mathrm{C}$ ) with tetrarhodamine isothiocyanate (TRITC)-rabbit anti-mouse conjugate (diluted 1:250), BrdU-incorporating cells were visualized by fluorescence microscopy and counted in 10 randomly chosen fields. (b) Cells growing in 24-well plates were exposed to toxin (quadruplicate samples) and postincubated in fresh medium as already described here. At the indicated times, the cells were incubated for $30 \mathrm{~min}$ with $\left[{ }^{3} \mathrm{H}\right]$ thymidine $(1 \mu \mathrm{Ci} / \mathrm{ml})$, and then rapidly removed by trypsinization and treated for $30 \mathrm{~min}$ with trichloroacetic acid (final concentration 10\%). The macromolecular fraction was precipitated by centrifugation $(13,000 \mathrm{~g} \times 10$ $\mathrm{min}$ ), washed in $10 \%$ trichloroacetic acid, dissolved in scintillation liquid (OptiPhase HiSafe; Fisons Chemicals, Loughborough, United Kingdom), and counted on a liquid scintillation counter (LKB Wallac, Loughborough, United Kingdom).Cell cycle analysis by flow cytometry. Cells were treated with $122 \mathrm{ng} / \mathrm{ml}$ HdCDT, rinsed with HBSS, scraped off (Cell Scraper; Nalge Nunc International, Naperville, Illinois, USA), and centrifuged $(3,500 \mathrm{~g} \times 7 \mathrm{~min})$. The pellet was resuspended in $1.5 \mathrm{ml}$ HBSS and fixed at $0^{\circ} \mathrm{C}$ for $10 \mathrm{~min}$ with $3 \mathrm{ml}$ ethanol $(70 \%)$. The cells were pelleted and stained for $15 \mathrm{~min}$ with $1 \mathrm{ml}$ propidium iodide (PI) solution $(0.05 \mathrm{mg} / \mathrm{ml}$; RNAse, $0.02 \mathrm{mg} / \mathrm{ml}$; NP40, $0.3 \%$; sodium citrate, $1 \mathrm{mg} / \mathrm{ml}$ ). Flow cytometry analysis of the DNA content was performed with a FACSort flow cytometer (Becton Dickinson Immunocytometry Systems, San Jose, California, USA). The data from $10^{3}$ cells were collected and analyzed using CellQuest software (Becton Dickinson).

Western blot. Cells were lysed in $300 \mu \mathrm{l}$ of SDS electrophoresis sample buffer (26) and samples were boiled for $10 \mathrm{~min}$. The proteins were separated by $10 \%$ SDS-PAGE, transferred to Protran Nitrocellulose (Schleicher \& Schuell, Dassel Germany) and probed with antiphosphotyrosine (Upstate Biotechnology) or anti-cdc2 (Transduction Laboratories, Lexington, Kentucky, USA) antibodies. Blots were developed with a Chemiluminescence Western Blotting Kit (Boehringer Mannheim, Mannheim, Germany) using a peroxidase-labeled secondary antibody. The amount of protein in the lysates was determined by Bio-Rad Protein Assay (Bio-Rad Laboratories, Hercules, California, USA) using BSA as a standard.

Two-dimensional gel electrophoresis. Cells were lysed in sample buffer (26), boiled for $10 \mathrm{~min}$, cooled on ice for $5 \mathrm{~min}$, and treated for $8 \mathrm{~min}$ at $22^{\circ} \mathrm{C}$ with a 1:20 diluted solution of DNAse I (1 $\mathrm{mg} / \mathrm{ml}$ ), RNAse (0.25 mg/ml), $0.5 \mathrm{M}$ Tris, $0.1 \mathrm{M} \mathrm{MgCl}_{2}$ (pH 7.0). Lysates were incubated $20 \mathrm{~min}$ at $22^{\circ} \mathrm{C}$ with acetone (final concentration $80 \%)$ and centrifuged $(12,000 \mathrm{~g} \times 10 \mathrm{~min})$. The pellet was washed once with acetone, centrifuged, dried for $5 \mathrm{~min}$, and resuspended in $50 \mu \mathrm{l}$ of buffer $(9.9 \mathrm{M}$ urea; $4 \%$ Triton X-100; $2.2 \%$ ampholytes; $100 \mathrm{mM} 2$-mercaptoethanol). After isoelectric focusing in 11-cm Immobiline dry strips ( $\mathrm{pH} 3-10$; Pharmacia Biotech $\mathrm{AB}$, Sollentuna, Sweden), the proteins were separated in the second dimension by $10 \%$ SDS-PAGE, transferred to nitrocellulose, and probed with anti-phosphotyrosine or anti-cdc2 antibodies.

Assay of cell proliferation and cell death. Cells were treated with HdCDT for 15 min on ice or continuously at $37^{\circ} \mathrm{C}$. At different time points, quadruplicates of control and treated cells were removed by trypsinization and counted. Cell death was assayed by uptake of trypan blue $(0.025 \%)$.

Immunoprecipitation of $p 34$ cdc2 . HEp-2 cells in $75-\mathrm{cm}^{2}$ flasks were treated with HdCDT for $15 \mathrm{~min}$ on ice followed by medium for $24 \mathrm{~h}$. Control cells were treated with nocodazole (100 $\mathrm{nM}$ ) for $16 \mathrm{~h}$ or untreated. The $\mathrm{p} 34^{\mathrm{cdc} 2}$ was immunoprecipitated using the Immunoprecipitation Kit (Protein G) (Boehringer Mannheim) with $4.5 \mu \mathrm{g}$ of cdc2 antibody and incubation for 5
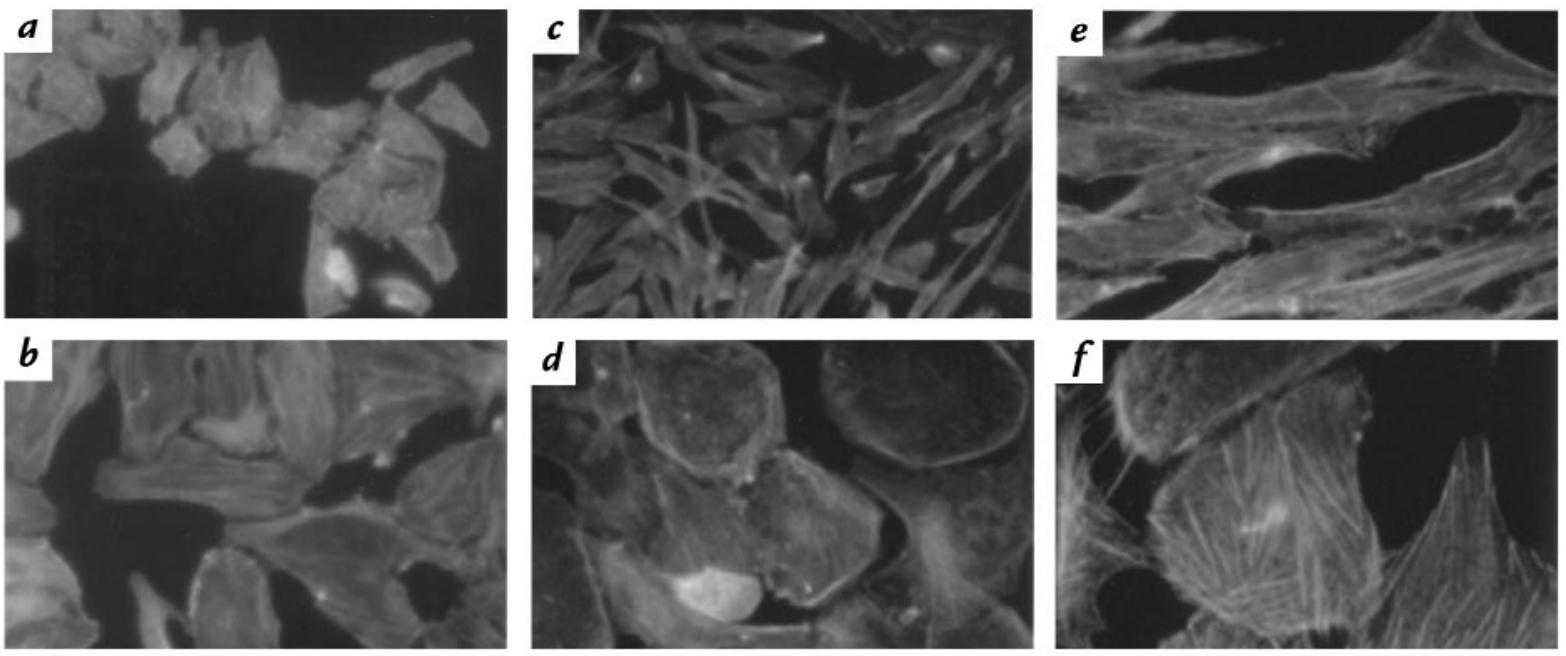

Figure 3

Effect of HdCDT on the ACSK of HEp-2 and Don cells. HEp-2 cells ( $\boldsymbol{a}$ and $\boldsymbol{b}$ ) were treated with toxin ( 15 min on ice) and postincubated at $37^{\circ} \mathrm{C}$ for $48 \mathrm{~h}$. Don cells $(\boldsymbol{c}-f)$ were exposed to HdCDT for $24 \mathrm{~h}$ at $37^{\circ} \mathrm{C}$. F-actin was stained with FITC-phalloidin as detailed in Methods. HEp- 2 cells: control $(a)$ and toxin treated $(b)$; Don cells: controls $(c, e)$ and toxin treated $(d, f) . \times 400(a-d) ; \times 1000(e$ and $f)$. ACSK, actin cytoskeleton. 


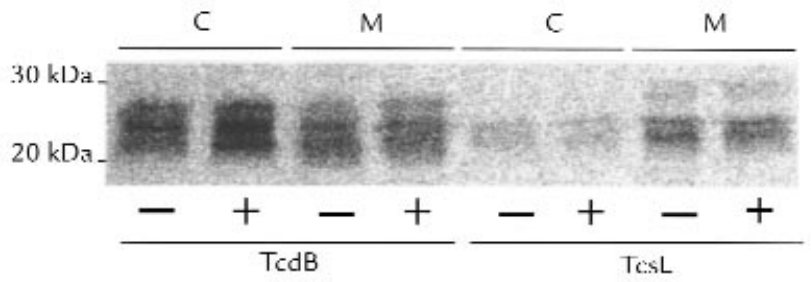

Figure 4

Effect of HdCDT on small GTP-binding proteins. HEp-2 cells were treated with $\mathrm{HdCDT}\left(15 \mathrm{~min}\right.$ on ice) and postincubated at $37^{\circ} \mathrm{C}$ for $24 \mathrm{~h}$. Lysates were prepared from these cells $(+)$ and control cells $(-)$. Cytosolic $(C)$ and membrane $(M)$ fractions were prepared by differential centrifugations and incubated $\left(1 \mathrm{~h}\right.$ at $\left.37^{\circ} \mathrm{C}\right)$ with TcdB or TcsL in the presence of UDP$\left[{ }^{14} \mathrm{C}\right]$ Glucose. Proteins were separated by $12.5 \%$ SDS-PAGE, and radiolabeled bands were detected by Phosphorlmager analysis. TcdB, Toxin B from Clostridium difficile; TcsL, Lethal Toxin from Clostridium sordellii.

$\mathrm{h}$ with the protein G-agarose beads. Samples were analyzed by Western blot with anti-phosphotyrosine antibodies.

Assay of histone H1 kinase activity. P34cdc2 in HEp-2 cell lysates $\left(1,500 \mu \mathrm{g}\right.$ protein) was immunoprecipitated. The $\mathrm{p} 34^{\mathrm{cdc} 2} / \mathrm{cyclin}$ $\mathrm{B}$ complexes were incubated $10 \mathrm{~min}$ at $37^{\circ} \mathrm{C}$ with $2.5 \mu \mathrm{Ci}$ of $[\gamma-$ $\left.{ }^{32} \mathrm{P}\right], 10 \mu \mathrm{g}$ of histone $\mathrm{H} 1$ (Boehringer Mannheim), $100 \mathrm{mM}$ $\mathrm{NaCl}, 10 \%$ Triton $\mathrm{X}-100,50 \mu \mathrm{M}$ ATP, and $10 \mathrm{mM} \mathrm{MgCl}_{2}$. Then, $10 \%$ SDS-PAGE radiolabeled bands were analyzed using a PhosphorImager SF (Molecular Dynamics).

\section{Results}

Sensitivity of different cell lines to HdCDT and optimal conditions for toxin treatment. A 15-minute exposure of precooled HEp-2 or HeLa cells to ice-cold HdCDT, followed by fresh toxin-free growth medium, sufficed to induce a morphological change in 12-24 hours, later resulting in cell death. Also the human keratinocyte line $\mathrm{HaCat}$ was sensitive (Fig. 1), although in this case, a two-hour incubation with HdCDT at $37^{\circ} \mathrm{C}$ was needed before the postincubation in medium. A requisite for the $\mathrm{CPE}$ in Don fibroblasts was the continued presence of HdCDT in the medium. Mouse 3T3 fibroblasts, however, did not change morphology even when treated continuously with HdCDT. Most experiments presented in the following section were performed on HEp-2 cells, using the 15-minute binding step on ice with $122 \mathrm{ng} / \mathrm{ml} \mathrm{HdCDT}$. These standard conditions were optimal for obtaining a reproducible CPE, beginning 12-15 hours after toxin treatment.

HdCDT-treated cells are enlarged, followed by shrinkage and deterioration. The first morphological change seen in toxin-sensitive cells was an enlargement. In $\mathrm{HaCat}$ cells, this was clear 24 hours after treatment with 122 $\mathrm{ng} / \mathrm{ml}$ (Fig. 1b) and cells rounded up after 48 hours (Fig. 1a). In HEp-2 and HeLa cells, a distention was already obvious 15 hours after toxin binding and maximal in 72 hours. After 24 hours, HEp-2 cells were three times, and after 72 hours five times, larger than the average control cell (Fig. 2). About 48 hours after toxin exposure, cells began to round up and shrink. Such cells incorporated trypan blue, indicating a damaged plasma membrane. Don fibroblasts were maximally enlarged after 24 hours of continued HdCDT treatment $(122 \mathrm{ng} / \mathrm{ml})$ and were completely destroyed without rounding after a total exposure time of 48 hours (data not shown).
The ACSK is promoted in HdCDT-treated cells. Another member of the CDT family was shown to induce actin rearrangements in Chinese hamster ovary (CHO) cells (27). Similar changes of F-actin occurred in HdCDTtreated cells. Prominent stress fibers developed and membrane ruffling was promoted in both HEp-2 and Don cells (Fig. 3, b, d, and f). Thus, the earliest morphological effect of HdCDT on these cells is a distention appearing concomitantly with a promoted ACSK.

Given that small GTPases control both the ACSK (28) and cell proliferation (29), we asked whether HdCDT might affect such proteins. For detection of small GTPases, we used two glucosyltransferase toxins, TcdB and TcsL, produced by C. difficile and C. sordellii, respectively, and known to glucosylate small GTPases of the Rho and Ras subfamilies (24). Lysates made of HdCDT-treated and maximally enlarged HEp-2 cells were mixed with each glucosyltransferase in the presence of UDP- $\left[{ }^{14} \mathrm{C}\right] \mathrm{glu}-$ cose (Fig. 4). Three observations suggested that HdCDT had no effect on small GTPases: (a) no alteration was detectable in the electrophoretic mobilities of any of the GTPases glucosylated by either TcdB or TcsL; (b) the extent of GTPase glucosylation by TcdB/TcsL was not altered in HdCDT-intoxicated cells; and (c) the distribution between the cytosolic and membrane fractions of
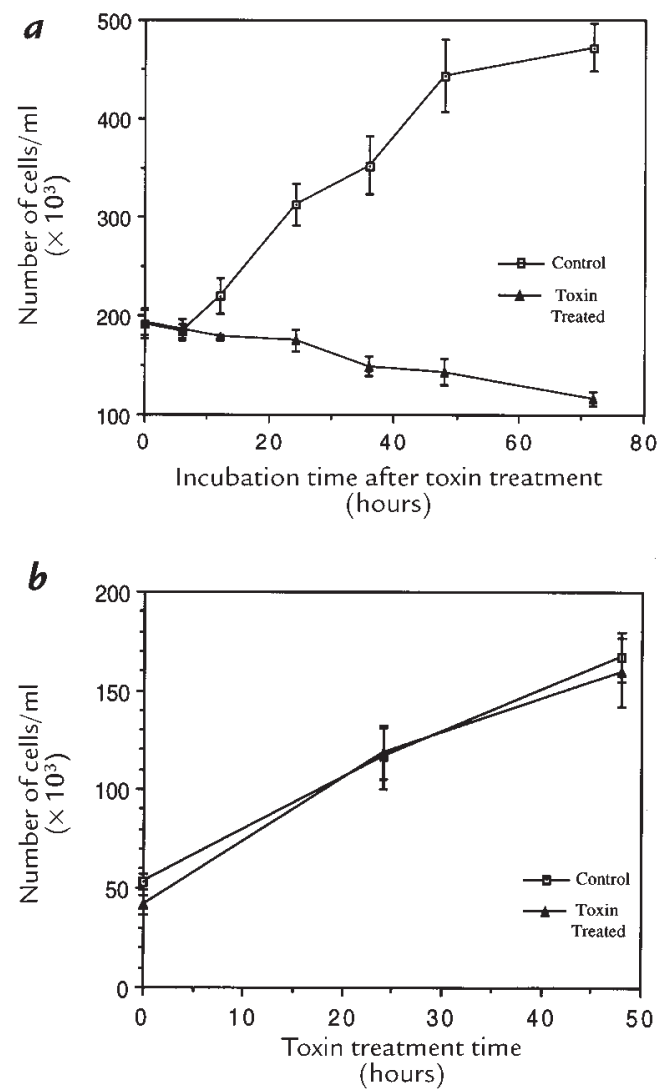

Figure 5

Effect of HdCDT on cell proliferation. (a) HEp-2 cells were treated with $\operatorname{HdCDT}$ (15 min on ice), postincubated at $37^{\circ} \mathrm{C}$ for the times indicated, trypsinized, and counted. (b) $3 \mathrm{~T} 3$ cells were treated with $\mathrm{HdCDT}$ at $37^{\circ} \mathrm{C}$ continuously until trypsinization and counting at the times indicated. Each point represents the mean of quadruplicate samples $\pm S D$. This is a representative experiment of two experiments, each of which was conducted with quadriplicate samples. 
$a$

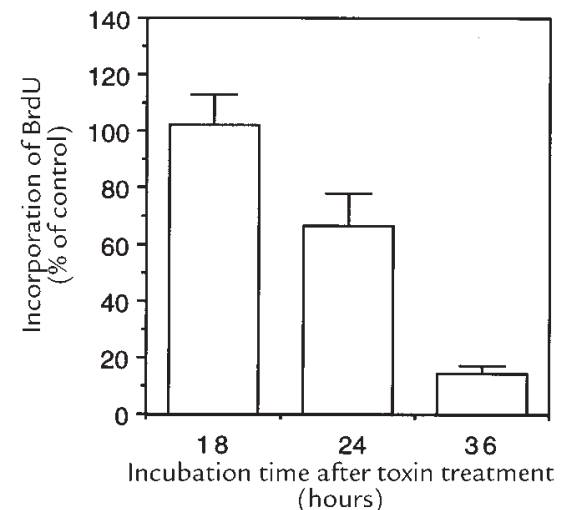

$\boldsymbol{b}$

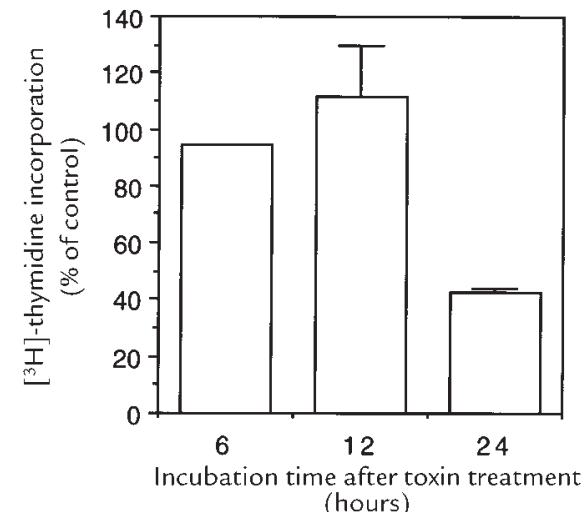

Figure 6

DNA synthesis in HdCDT-treated cells. (a) Incorporation of BrdU. HEp2 cells treated with HdCDT for $15 \mathrm{~min}$ on ice were postincubated for 18,24 , and $36 \mathrm{~h}$ in medium at $37^{\circ} \mathrm{C}$. BrdU $(10 \mu \mathrm{g})$ was present during the final $12 \mathrm{~h}$, and its incorporation was visualized by staining with anti-BrdU. (b) Incorporation of $\left[{ }^{3} \mathrm{H}\right]$ thymidine. HEp-2 cells treated with HdCDT as described above were postincubated for 6,12 , and $24 \mathrm{~h}$. $\left[{ }^{3} \mathrm{H}\right]$ thymidine $(1 \mu \mathrm{Ci} / \mathrm{ml})$ was present during the final $30 \mathrm{~min}$, and its incorporation in DNA was determined as detailed in Methods. Mean values + SD of three different experiments. BrdU, bromodeoxyuridine.

GTPases glucosylated by these toxins was not changed. $H d C D T$ lacks ADP-ribosyltransferase or glucosyltransferase activities. Because ADP ribosylation is a common effect of many bacterial toxins, we investigated whether HdCDT would ADP ribosylate some cellular target protein. HEp2 cell lysates were treated with HdCDT in the presence of [ $\left.{ }^{32} \mathrm{P}\right] \mathrm{NAD}$ according to a protocol described previously (24) and using C. botulinum exoenzyme $\mathrm{C} 3$ as positive control. However, no ADP-ribosylated proteins were detectable in such treated cell lysates (data not shown). HdCDT was also devoid of glucosyltransferase activity as tested in a principally similar way (25), but using UDP- $\left[{ }^{14} \mathrm{C}\right]$ glucose as the cofactor and C. difficile $\mathrm{TcdB}$ as the positive control (data not shown). Thus, none of these two enzymatic activities can be responsible for the cytotoxic effect induced by HdCDT.

$H d C D T$ inbibits cell proliferation. We determined the capacity of HdCDT-treated cells to proliferate by counting them at various stages of intoxication. After the brief standard exposure to HdCDT, the HEp-2 cells apparently did not divide at all (Fig. 5a). The same effect was induced in HaCat, HeLa, and Don cells (data not shown). By contrast, 3T3 fibroblasts proliferated normally despite the continuous presence of HdCDT in the medium (Fig. 5b), consistent with the finding that these cells were not morphologically altered by the toxin. The synthesis of DNA in HdCDT-exposed HEp-2 cells was not affected up to 18 hours after treatment, and incorporation of both BrdU and $\left[{ }^{3} \mathrm{H}\right]$ thymidine was decreased 24-36 hours after toxin exposure (Fig. 6). Thus, the division of cells responding to HdCDT with a CPE was irreversibly inhibited, but a blocked DNA synthesis was not the primary cause of this antiproliferative effect .

HdCDT-treated cells are arrested in G2 phase. Flow cytometric analysis of HdCDT-treated HEp-2 cells indicated a block in the G2/M phase (Fig. 7), similar as described for other CDTs $(15,20)$. The nuclei were enlarged and no mitotic figures were seen, suggesting that the cells were blocked in the G2 rather than the M phase.

p34cdc2 in HdCDT-treated cells remains tyrosine phosphorylated and inactive. A hyperphosphorylation of the p34 cdc2 kinase was demonstrated in cells treated with other CDTs $(20,21)$. We probed the proteins in HdCDT-treated cells by Western blot with anti-phosphotyrosine antibodies. Indeed $\mathrm{p} 34^{\mathrm{cdc} 2}$ was significantly more phosphorylated than in control lysates (data not shown). The p34cdc2 protein was immunoprecipitated and its kinase activity was measured. In toxin-treated cells, the p34cdc2 kinase activity was about $20 \%$ of that in cells synchronized into the G2/M phase by treatment with nocodazole (Fig. 8a). Immunoprecipitated $\mathrm{p} 34^{\mathrm{cdc} 2}$ was visualized with antiphosphotyrosine antibody (Fig. 8b). As expected, the p34 cdc2 in nocodazole-treated cells showing a high kinase

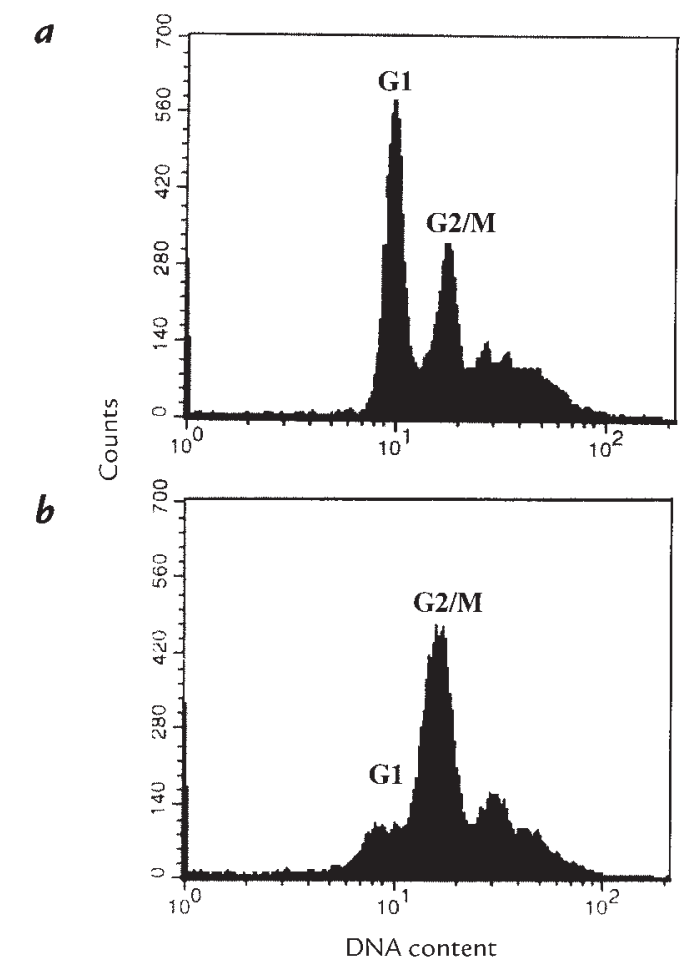

\section{Figure 7}

Flow cytometric analysis of HdCDT-treated HEp-2 cells. DNA was stained with propidium iodide. (a) Control cells. (b) Cells exposed to HdCDT (15 min on ice) and postincubated for $36 \mathrm{~h}$. 
activity was not tyrosine phosphorylated, whereas that in the toxin-treated cells was (Fig. 8b). Although the p34 cdc2 in untreated control cells was also not phosphorylated, its activity (Fig. 8a) was as low as in the HdCDT-treated cells, because most of these cells were in interphase; i.e., the $\mathrm{p} 34^{\mathrm{cdc}} /$ cyclin complex was not yet formed.

The phosphorylation of $\mathrm{p} 34^{\mathrm{cdc} 2}$ was already increased six hours after toxin exposure, as judged both by the labeling intensity of the protein and the fact that it migrated somewhat more slowly than that in control cell lysates (Fig. 9a). By contrast, the HdCDT-insensitive 3T3 fibroblasts showed no change in tyrosine phosphorylation of $\mathrm{p} 34^{\mathrm{cdc} 2}$ (Fig. 9b), indicating that the observed phosphorylation was indeed related to the cytotoxicity. A two-dimensional separation of the proteins in HEp-2 cell lysates identified two proteins reacting with antip34cdc2 antibodies in control cells (Fig. 10c). In lysates from HdCDT-treated cells, three new spots appeared in the more acidic part of the gel (Fig. 10b). Two of these were significantly tyrosine phosphorylated and migrated more slowly than the unphosphorylated forms (Fig. 10a). We conclude that HdCDT treatment prevents the exit of cells from the G2 phase, because their p34 cdc2 remains inactive owing to tyrosine phosphorylation.

\section{Discussion}

Our long-term aim is to understand the pathophysiology behind the $H$. ducreyi infection by investigating the molecular actions of putative virulence factors of this bacterium (30). Here we show that the CDT purified from $H$. ducreyi, like other members of the CDT family $(15,20,21)$, irreversibly blocks mammalian cell proliferation by inducing cell-cycle arrest in G2.

Sensitive cells and receptor. We reported previously (10) that HdCDT was able to intoxicate human epithelioid cell lines. We now show that the spectrum of toxin-sensitive cells is broader, including a human keratinocyte line as well as hamster lung fibroblasts. In addition, we have recently observed that normal human monocytes, $\mathrm{T}$ and $B$ cells, as well as normal human epidermal keratinocytes and fetal human fibroblasts, are sensitive to HdCDT (Svensson, L., and Lagergård, T., manuscript submitted for publication). The intoxication of HEp-2 and HeLa cells was irreversible after a short toxin exposure on ice, allowing binding but not internalization of the toxin. We conclude that these cells probably expose a high-affinity receptor for HdCDT. The receptor may exist in lower numbers or in a low-affinity form in Don fibroblasts , which were intoxicated only upon continued toxin exposure at $37^{\circ} \mathrm{C}$, suggesting they could not bind HdCDT rapidly at low temperature. Although we do not know why $3 \mathrm{~T} 3$ cells resist intoxication by HdCDT, we speculate that the putative toxin-specific receptor might be completely absent from 3 T3 cells. HEp-2 cells will constitute a suitable model system in future identification of the cell-surface receptor for HdCDT.

Cell distention and effects on the ACSK. The effect of E. coli $\mathrm{CDT}$ on the ACSK of CHO cells was speculated to result from a toxin attack on Rho proteins (27). We believe that the ACSK-promoting effect, by HdCDT at least, may be mediated by some signaling system other than the Rho proteins, because none of our experiments supported their direct involvement. The ACSK is controlled by Rho, Rac, and Cdc42 (28), which are targets of TcdB, whereas Ras, Rap, Ral, and Rac are attacked by TcsL $(31,32)$. Both TcdB and TcsL were able to fully glucosylate all their GTPase substrates in lysates from HdCDT-treated HEp-2 cells, and all these GTPases had unchanged electrophoretic mobilities. We previously detected a mobility change of ADP-ribosylated Rho by glucosylation with TcdB (Fig. $8 a$ in ref. 25). Moreover, Rho in lysates from cells pretreated with TcdB could not be ADP ribosylated $(25,33)$. By analogy, our present observations suggest that HdCDT does not covalently modify the effector region of any of the GTPases attacked by TcdB and TcsL. In view of the similarities in action between the known CDTs, these observations may be valid also for the other toxins in this family.

The ACSK promotion and enlargement of cells exposed to HdCDT or other CDTs resemble the effects of the cytotoxic necrotizing factor (CNF) from E. coli. CNF permanently activates Rho by deamidation of its Gln-63 $(34,35)$. This effect would not be detectable as a changed electrophoretic mobility of Rho. However,

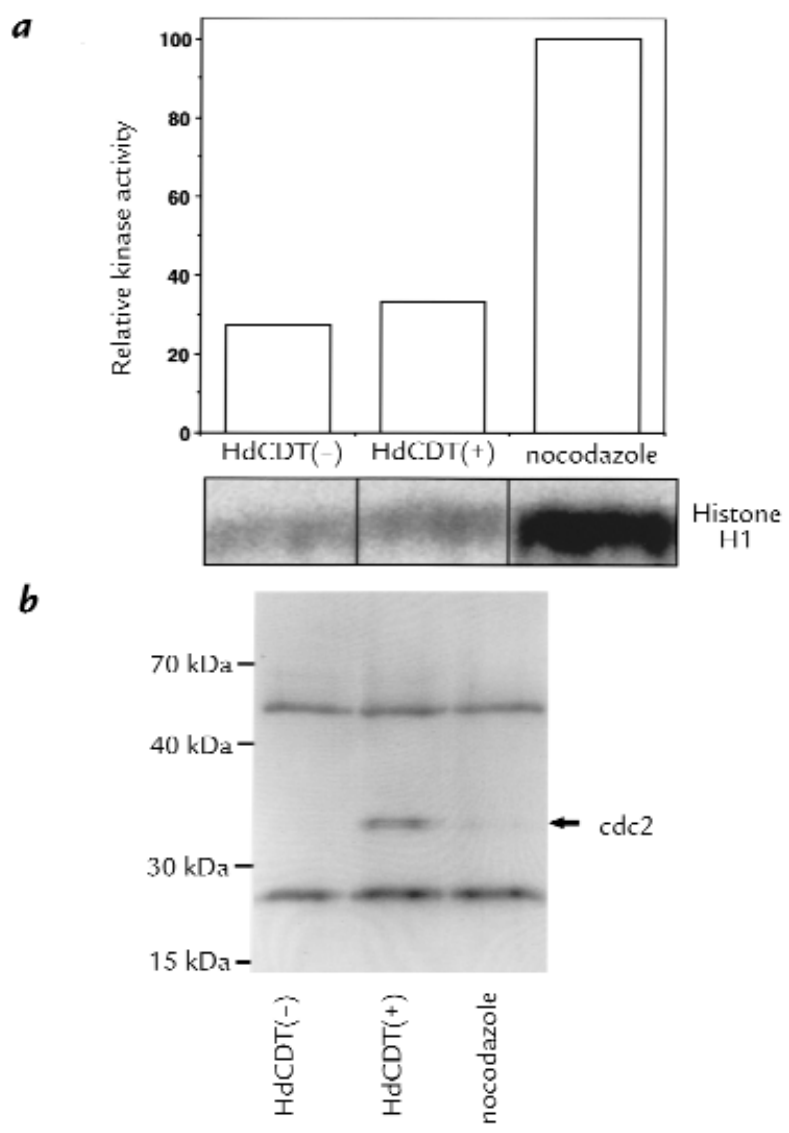

\section{Figure 8}

Protein kinase activity of immunoprecipitated $\mathrm{p} 34^{\mathrm{cdc} 2}$ from HdCDT-treated cells. (a) HEp-2 cells were exposed to toxin (15 min on ice), postincubated for $24 \mathrm{~h}$, and lysed. The p34cdc2 was immunoprecipitated and its kinase activity toward histone $\mathrm{H} 1$ was determined. Controls: lysates from nocodazole-treated cells and from nontreated interphase cells. (b) Western blot of the immunoprecipitated $\mathrm{p} 34^{\mathrm{cd} c 2}$ with anti-phosphotyrosine antibodies. The two bands flanking the $p 34^{\mathrm{cdc} 2}$ represent the heavy and light chains of the antibody used for immunoprecipitation that have reacted with the secondary antibody. 

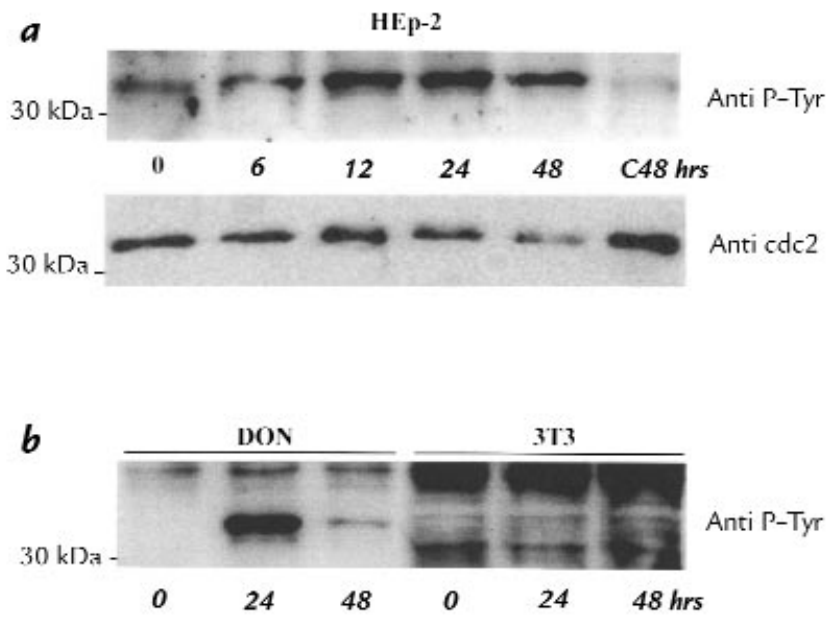

Figure 9

Tyrosine phosphorylation of p $34^{\text {cdc } 2}$ in HdCDT-treated cells. (a) HEp-2 cells were exposed to the toxin $\left(15 \mathrm{~min}\right.$ on ice) and postincubated at $37^{\circ} \mathrm{C}$. After the times indicated, cells were processed for Western blot with anti-phosphotyrosine or anti-cdc2 antibodies. The same sample was used for both immunoblots. (b) Don and $3 \mathrm{~T} 3$ cells were treated with $\mathrm{HdCDT}$ at $37^{\circ} \mathrm{C}$ for 24 and $48 \mathrm{~h}$ and probed with anti-phosphotyrosine antibodies.

CNF-treated HEp-2 cells are resistant to the ACSK collapsing effect of TcdB (36), whereas the HdCDT-treated cells remained fully sensitive to $\mathrm{TcdB}$ (data not shown). Furthermore, multinucleation occurs in a majority of cells intoxicated by CNF (15) but was rarely seen in cells treated with HdCDT (this paper) or E. coli CDT (15). Thus, a similar effect on Rho as by CNF appears unlikely for HdCDT, as was also concluded for CDT (15). Finally, we considered the possibility that HdCDT could activate small GTPases indirectly by promoting their recruitment to the plasma membrane (28), but this hypothesis was also rejected (Fig. 4). Although we cannot exclude a very subtle HdCDT-induced novel type of small GTPase modification, undetectable by gel electrophoresis, a secondary ACSK-affecting signal from the nucleus appears more likely (see later here).

Effect on the cell-cycle machinery. HdCDT-treated cells accumulated in the G2 phase, explaining why they could not proliferate at all, and consistent with the hypothesis that the cyclin-dependent kinase required for G2-to-M transition might be a target of the toxin. In cells cycling normally, this $\mathrm{p} 34^{\mathrm{cdc} 2}$ kinase is activated when a phosphatase (Cdc25) removes the inhibitory phosphates from Thr-14 and Tyr-15 (22). In HdCDT-intoxicated cells, we observed an increased tyrosine phosphorylation of $\mathrm{p} 34^{\mathrm{cdc} 2}$. It was also shown to have a significantly lower kinase activity than the p34 $4^{\mathrm{cdc} 2}$ in nocodazole-treated positive control cells (Fig. 8). In the study demonstrating that the three CDT variants of $E$. coli prevent $\mathrm{p} 34^{\mathrm{cdc} 2}$ dephosphorylation in HeLa cells (20), this effect was determined 24 hours after toxin exposure. The HdCDT effect was already detectable within six hours after toxin exposure (Fig. 9), hours before any significant signs of cell distention and ACSK promotion. The same phenotypic effects on the cell cycle have been recently documented in cells infected with HIV or transfected with $v p r$, one of its regulatory genes (37-40).
The currently known mechanisms for G2 arrest point to several possible explanations for a $\mathrm{p} 34^{\text {cdc2 }}$ hyperphosphorylation. It could be due to (a) damage of DNA or inhibition of DNA synthesis, evoking the G2 checkpoint response $(41,42)$; (b) hyperactivation of the kinases responsible for phosphorylation of p34 $4^{\text {cdc2 }}$ at Thr-14 and Tyr-15 (22); (c) a lack of activation or an inactivation of the Cdc25 phosphatase, which dephosphorylates at these positions (43); or even (d) a direct inactivation of p34 $4^{\text {cdc2 }}$, which might result in its hyperphosphorylation (44). As the hyperphosphorylated p34cdc2 in HdCDT-treated cells appeared (Fig. 9) many hours before detectable failure to synthesize DNA (Fig. 6), an impaired DNA synthesis does not appear to be a primary effect of the toxin. Indeed, we found recently that the p34cdc2 in completely confluent monolayers of Don fibroblasts was also tyrosine phosphorylated upon HdCDT exposure, despite the fact that these cells were not dividing (data not shown). A primary slight damage to DNA, however, cannot be excluded at this stage. The mechanism of these effects by other CDTs and by $v$ pr are also not known, but at least E. coli CDT (27) had no early effect on the synthesis of DNA, whereas results on $v p r$ are contradictory $(39,40)$. The possibility that HdCDT acts directly on p34cdc2 or on some of the kinases/phosphatases in the signaling network controlling its activity should be explored.

The connection between the G2 arrest and the cell enlargement, with promoted ACSK induced by CDTs, is not presently understood. From the severalfold increase in cell area, it is clear that toxin-treated cells continue to grow after the $\mathrm{G} 2$ arrest. One (remote) possibility is that the CDTs could harbor two different cytotoxic activities, one affecting the cell-cycle machinery and another affect-

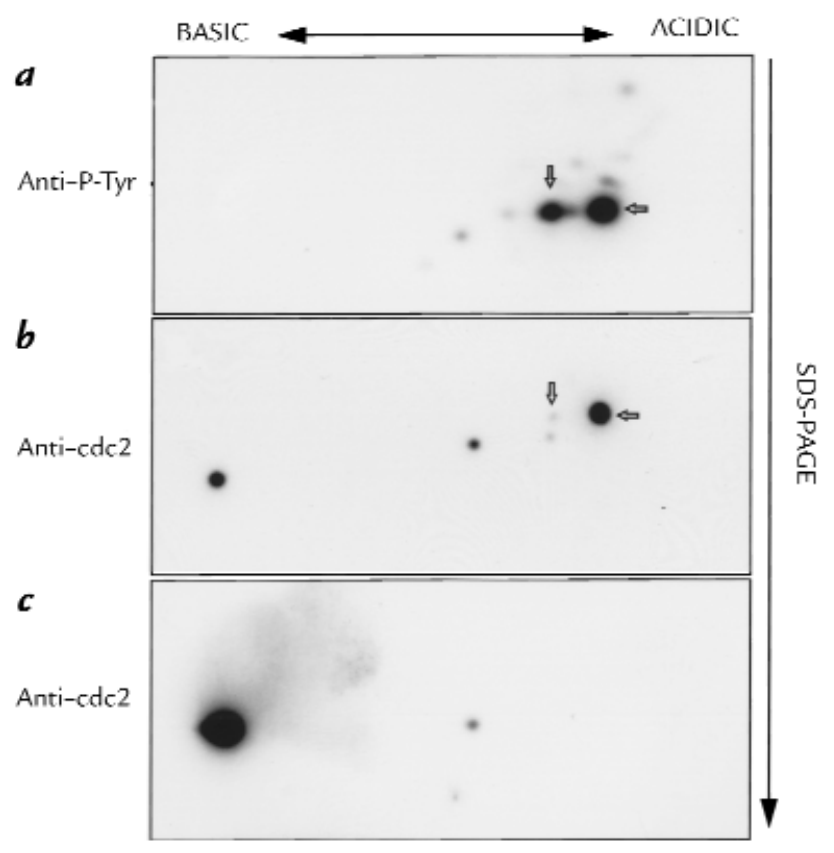

Figure 10

Two-dimensional gel electrophoresis of p34cdc2 in HdCDT-treated cells. HEp-2 cells were exposed to the toxin (15 min on ice), postincubated for $48 \mathrm{~h}$, and lysed. Proteins in the cell lysates were separated in two dimensions (see Methods) and analyzed by Western blot as for Fig. 9. 
ing the ACSK. A similar severalfold enlargement of cells arrested in G2 occurred upon transfection with the $v p r$ gene $(37,38)$. In the light of this finding, it appears more plausible that the enlargement induced by HdCDT and other CDTs is linked with, but secondary to, the molecular events leading to $\mathrm{G} 2$ arrest.

Clinical considerations. CDT encoded in E. coli by the Shigella cdt genes caused secretory diarrhea and necrosis of the colonic epithelium in suckling mice (45), as demonstrated with a CDT- isogenic mutant. However, the relevance of CDT in human infections is far from clear and is not a trivial problem to elucidate, because CDT-producing E. coli strains may elaborate several toxins and may cause both gastrointestinal and extraintestinal infections $(13,16,46)$. HdCDT, on the other hand, is one of two described toxins produced by $H$. ducreyi. The hemolysin (7) is active mainly on foreskin fibroblasts and does not affect epithelial cells or human skin (9). In contrast, HdCDT kills epithelial cells, and this potent toxin is produced by a majority of $H$. ducreyi isolates (47). Serum antibodies neutralizing the HdCDT were detected at significantly higher levels in patients with culture-confirmed chancroid than in healthy blood donors, indicating production of the toxin during the disease (12). Moreover, different strains of $H$. ducreyi cause one and the same disease, characterized by destruction of epidermis, and to some degree, the dermis.

HdCDT could be involved in genital ulceration by multiple actions. Given that the toxin blocks cell proliferation, it is likely to exert the strongest effect on cells of the basal layer of the epidermis, which need to constantly proliferate. The early events in the pathogenesis of chancroid may then involve minor abrasions of the epidermis, adherence of infecting bacteria to epidermal cells, and bacterial multiplication and production of HdCDT (48). A small amount of HdCDT can destroy both keratinocytes and other epithelial cells, thus providing a highly nourishing environment for the continued growth of this fastidious bacterium, facilitating enlargement of the ulcer. H. ducreyi is relatively resistant to the serum bactericidal activity (49), a feature that also should contribute to the persistence of bacteria in the lesions. When the ulcer progresses more deeply, fibroblasts of the dermis can be attacked by the toxin. At this stage, the hemolysin could aggravate the lesion, causing progression in the dermis. This is consistent with observations that chancroid ulcers are well vascularized and bleed easily on manipulation (50). The irreversible inhibition of cell proliferation by HdCDT may also explain the retardation of healing that is characteristic of chancroid. In fact, before the era of antibiotic treatment, the giant chancre in some patients could persist for months (50).

In conclusion, we have shown that HdCDT, the potent CDT produced by H. ducreyi, like other CDTs inhibits cell proliferation by G2 arrest because the p34cdc2 kinase remains tyrosine phosphorylated and therefore inactive. The enzymatic (?) mode of action of the CDTs is not known, but for HdCDT we have excluded ADP-ribosyltransferase and glucosyltransferase activities. The irreversible block of cell proliferation is consistent with the generation of ulcers as well as their slow healing. In all probability, HdCDT is the most important virulence fac- tor in ulcer formation by $H$. ducreyi. Moreover, HdCDT and other CDTs may become interesting new tools for studies of the signal transduction events in the transition of mammalian cells from the G2 phase into mitosis.

\section{Acknowledgments}

We thank C. von Eichel-Streiber for large clostridial cytotoxins, and T. Frisan (Microbiology and Tumorbiology Center, Karolinska Institutet, Stockholm, Sweden) for advice concerning flow cytometry. This work was supported by the Swedish Medical Research Council (05969), Karolinska Institutet, and Swedish International Development Cooperation Agency, Department for Research Cooperation. X. Cortes-Bratti was supported by a fellowship from the Swedish Institute.

1. Plummer, F.A., et al. 1983. Epidemiology of chancroid and Haemophilus ducreyi in Nairobi, Kenya. Lancet. 2:1293-1295.

2. Morse, S.A. 1989. Chancroid and Haemophilus ducreyi. Clin. Microbiol. Rev. 2:137-157.

3. Trees, D.L., and Morse, S.A. 1995. Chancroid and Haemophilus ducreyi: an update. Clin. Microbiol. Rev. 8:357-375.

4. Schryver, A. De, and Maheus, A. 1990. Epidemiology of sexually transmitted diseases: the global picture. Bull. World Health Organ. 68:639-654.

5. Campagnari, A.A., et al. 1991. Role of lipo-oligosaccharide in experimental dermal lesions caused by Haemophilus ducreyi. Infect. Immun. 59:2601-2608.

6. Lagergård, T. 1992. The role of Haemophilus ducreyi bacteria, cytotoxin, endotoxin and antibodies in animal models for study of chancroid. Microb. Pathog. 13:203-217.

7. Palmer, L., and Munson, R.S., Jr. 1995. Cloning and characterization of the genes encoding the hemolysin of Haemophilus ducreyi. Mol. Microbiol. 18:821-830.

8. Totten, P., Norn, D.V., and Stamm, W.E. 1995. Characterization of the hemolytic activity of Haemophilus ducreyi. Infect. Immun. 63:4409-4416.

9. Palmer, K.L., et al. 1998. Evaluation of a isogenic hemolysin-deficient mutant in the human model of Haemophilus ducreyi infection. J. Infect. Dis. 178:191-199.

10. Purvén, M., and Lagergård, T. 1992. Haemophilus ducreyi, a cytotoxin-producing bacterium. Infect. Immun. 60:1156-1162.

11. Lagergård, T., and Purvén, M. 1993. Neutralizing antibodies to Haemophilus ducreyi cytotoxin. Infect. Immun. 61:1589-1592.

12. Purvén, M., Frisk, A., Lönnroth, I., and Lagergård, T. 1997. Purification and identification of Haemophilus ducreyi cytotoxin using a neutralizing monoclonal antibody. Infect. Immun. 65:3496-3499.

13. Scott, D.A., and Kaper, J.B. 1994. Cloning and sequencing of the genes encoding Escherichia coli cytolethal distending toxins. Infect. Immun. 62:244-251.

14. Pickett, C.L., Cottle, D.L., Pesci, E.C., and Bikah, G. 1994. Cloning, sequencing, and expression of the Escherichia coli cytolethal distending toxin genes. Infect. Immun. 62:1046-1051.

15. Pérès, S.Y., et al. 1997. A new cytolethal distending toxin (CDT) from Escherichia coli producing CNF2 blocks HeLa cell division in G2/M phase. Mol. Microb. 24:1095-1107.

16. Okuda, J., Kurazono, H., and Takeda, Y. 1995. Distribution of the cytolethal distending toxin A gene ( $c d t A)$ among species of Shigella and Vibrio and cloning and sequencing of the $c d t$ gene from Shigella dysenteriae. Microb. Pathog. 18:167-172.

17. Pickett, C.L., et al. 1996. Prevalence of cytolethal distending toxin production in Campylobacter jejuni and relatedness of Campylobacter sp $c d t B$ genes. Infect. Immun. 64:2070-2078.

18. Sugai, M., et al. 1998. The cell cycle-specific growth-inhibitory factor produced by Actinobacillus actinomycetemcomitans is a cytolethal distending toxin. Infect. Immun. 66:5008-5019.

19. Cope, L.D., et al. 1997. A diffusible cytotoxin of Haemophilus ducreyi. Proc. Natl. Acad. Sci. USA. 94:4056-4061.

20. Comayras, C., et al. 1997. Escherichia coli cytolethal distending toxin blocks the HeLa cell cycle at the $\mathrm{G}_{2} / \mathrm{M}$ transition by preventing cdc2 protein kinase dephosphorylation and activation. Infect. Immun. 65:5088-5095.

21. Whitehouse, C.A., et al. 1998. Campylobacter jejuni cytolethal distending toxin causes a G2-phase cell cycle block. Infect. Immun. 66:1934-1940.

22. Jackman, M.R., and Pines, J.N. 1997. Cyclins and the $G_{2} / M$ transition, Cancer Surv. 29:47-73.

23. Boukamp, P., et al. 1988. Normal keratinization in a spontaneously immortalised aneuploid human keratinocyte cell line. J. Cell. Biol. 106:761-771.

24. Chaves-Olarte, E., et al. 1996. UDP-glucose deficiency in a mutant cell line protects against glucosyltransferase toxins from Clostridium difficile and Clostridium sordellii.J. Biol. Chem. 271:6925-6932. 
25. Chaves-Olarte, E., Weidmann, M., Eichel-Streiber, C.V., and Thelestam, M. 1997. Toxins A and B from Clostridium difficile differ with respect to enzymatic potencies, cellular substrate specificities, and surface binding to cultured cells. J. Clin. Invest. 100:1734-1741.

26. Laemmli, U.K. 1970. Cleavage of structural proteins during the assembly of the head of bacteriophage T4. Nature. 227:680-685.

27. Aragon,V., Chao, K., and Dreyfus, L.A. 1997. Effect of cytolethal distending toxin on F-actin assembly and cell division in Chinese hamster ovary cells. Infect. Immun. 65:3774-3780.

28. Hall, A. 1994. Small GTP-binding proteins and the regulation of the actin cytoskeleton. Annu. Rev. Cell. Biol. 10:31-54.

29. Olson, M.F., Ashworth, A., and Hall, A. 1995. An essential role for Rho, Rac, and Cdc42 GTPases in cell cycle progression through G1. Science. 269:1270-1272.

30. Frisk, A., Ahmed, H.J., Dyck, E. Van, and Lagergård, T.1998. Antibodies specific to surface antigens are not effective in complement-mediating killing of Haemophilus ducreyi. Microb. Pathog. 25:67-75.

31. Eichel-Streiber, C.v., Boquet, P., Sauerborn, M., and Thelestam, M. 1996. Large clostridial cytotoxins - a family of glycosyltransferases modifying small GTP-binding proteins. Trends Microbiol. 4:375-382.

32. Hofmann, F., Rex, G., Aktories, K., and Just, I. 1996. The Ras-related protein Ral is monoglucosylated by Clostridium sordellii lethal toxin. Biochem. Biophys. Res. Commun. 227:77-81.

33. Just, I., et al. 1994. Clostridium difficile toxin B acts on the GTP-binding protein Rho. J. Biol. Chem. 269:10706-10712.

34. Schmidt, G., et al. 1997. Gln63 of Rho is deamidated by Escherichia col cytotoxic necrotizing factor-1. Nature. 387:725-729.

35. Flatau, G., et al. 1997. Toxin-induced activation of the G protein $\mathrm{p} 21$ Rho by deamidation of glutamine. Nature. 387:729-733.

36. Fiorentini, C., et al. 1995. Escherichia coli cytotoxic necrotizing factor 1: evidence for induction of actin assembly by constitutive activation of the p21 Rho GTPase. Infect. Immun. 63:3936-3944.

37. He, J., et al. 1995. Human immunodeficiency virus type 1 viral protein $\mathrm{R}$ $(v p r)$ arrests cells in the $\mathrm{G}_{2}$ phase of the cell cycle by inhibiting $\mathrm{p} 34^{\mathrm{cdc} 2}$ activity. J. Virol. 69:6705-6711.

38. Re, F., Braaten, D., Franke, E.K., and Luban, J. 1995. Human immuno deficiency virus type $1 v p r$ arrests the cell cycle in $\mathrm{G}_{2}$ by inhibiting the activation of $\mathrm{p}^{34} 4^{\mathrm{cdc} 2}$-cyclin B. J. Virol. 69:6859-6864.

39. Bartz, S.R., Rogel, M.E., and Emerman, M. 1996. Human immunodeficiency virus type 1 cell cycle control: $\nu p r$ is cytostatic and mediates $\mathrm{G}_{2}$ accumulation by a mechanism which differs from DNA damage checkpoint control. J. Virol. 70:2324-2331.

40. Poon, B., et al. 1997. Human immunodeficiency virus type $1 v p r$ gene induces phenotypic effects similar to those of the DNA alkylating agent, nitrogen mustard. J. Virol. 71:3961-3971.

41. Elledge, S.J. 1996. Cell cycle checkpoints: preventing an identity crisis. Science. 274:1664-1671.

42. O'Connell, M.J., Raleigh, J.M., Verkade, H.M., and Nurse, P. 1997. Chk1 is a wee 1 kinase in the $\mathrm{G}_{2}$ DNA damage checkpoint inhibiting cdc2 by Y15 phosphorylation. EMBOJ. 16:545-554.

43. Sanchez, Y., et al. 1997. Conservation of the Chk1 checkpoint pathway in mammals: linkage of DNA damage to cdk regulation through Cdc25. Science. 277:1497-1501.

44. Poon, R.Y., Chau, M.S., Yamashita, K, and Hunter, T. 1997. The role of cdc2 feedback loop control in the DNA damage checkpoint in mammalian cells. Cancer Res. 57:5168-5178.

45. Okuda, J., Fukumoto, M., Takeda, Y., and Nishibuchi, M. 1997. Examination of diarrheagenicity of cytolethal distending toxin: suckling mouse response to the products of the $c d t A B C$ genes of Shigella dysenteriae. Infect. Immun. 65:428-433.

46. Johnson, W.M., and Lior, H. 1988. A new heat-labile cytolethal distending toxin (CLDT) produced by Escherichia coli isolates from clinical material. Microb. Pathog. 4:103-113.

47. Purvén, M., Falsen, E., and Lagergård, T. 1995. Cytotoxin production in 100 strains of Haemophilus ducreyi from different geographic locations. FEMS Microbiol. Lett. 129:221-224.

48. Lagergård, T., Purvén, M., and Frisk, A. 1993. Evidence of Haemophilus ducreyi adherence to and destruction of human epithelial cells. Microb. Pathog. 14:417-431.

49. Lagergård, T., Frisk, A., Purvén, M., and Nilsson, L.Å. 1995. Serum bactericidal activity and phagocytosis in host defence against Haemophilus ducreyi. Microb. Pathog. 18:37-51.

50. Sullivan, M. 1940. Chancroid. American Journal of Syphilis. 24:482-521. 